\begin{tabular}{|c|l|}
\hline Title & Catalytic reaction dynamics in inhomogeneous networks \\
\hline Author(s) & Watanabe, A kitomo; Y akubo, Kousuke \\
\hline Citation & $\begin{array}{l}\text { Physical Review E, 89(5), 052806-1-052806-7 } \\
\text { https://doi.org/10.1103/PhysRevE.89.052806 }\end{array}$ \\
\hline Issue Date & 201405-12 \\
\hline Doc URL & http://hdl.handle.net/2115/57011 \\
\hline Rights & @2014 A merican Physical Society \\
\hline Type & article \\
\hline File Information & PhysRevE89 052806.pdf \\
\hline
\end{tabular}

Instructions for use 


\title{
Catalytic reaction dynamics in inhomogeneous networks
}

\author{
Akitomo Watanabe* and Kousuke Yakubo ${ }^{\dagger}$ \\ Department of Applied Physics, Hokkaido University, Sapporo 060-8628, Japan
}

(Received 24 December 2013; published 12 May 2014)

\begin{abstract}
Biochemical reactions in a cell can be modeled by a catalytic reaction network (CRN). It has been reported that catalytic chain reactions occur intermittently in the CRN with a homogeneous random-graph topology and its avalanche-size distribution obeys a power law with the exponent 4/3 [A. Awazu and K. Kaneko, Phys. Rev. E 80, 010902(R) (2009)]. This fact indicates that the catalytic reaction dynamics in homogeneous CRNs exhibits self-organized criticality (SOC). Structures of actual CRNs are, however, known to be highly inhomogeneous. We study the influence of various types of inhomogeneities found in real-world metabolic networks on the universality class of SOC. Our numerical results clarify that SOC keeps its universality class even for networks possessing structural inhomogeneities such as the scale-free property, community structures, and degree correlations. In contrast, if the CRN has inhomogeneous catalytic functionality, the universality class of SOC depends on how widely distributed the number of reaction paths catalyzed by a single chemical species is.
\end{abstract}

DOI: 10.1103/PhysRevE.89.052806

PACS number(s): 05.65.+b, 82.39.Rt, 89.75.-k

\section{INTRODUCTION}

Biochemical reactions in cells are necessary for life to survive. These reactions are catalyzed by functional proteins called enzymes supplied from extracellular fluid or synthesized by intracellular reactions. Products by such reactions are responsible for subsequent reactions or are exhausted to outside of cells. In order to extract universal statistical properties of complicated chain reactions, metabolic networks representing catalytic reaction paths in a cell have been extensively studied [1-18]. Most of these studies are classified into two types. One is focused on dynamical reaction processes in a network [1-5], which have been mainly studied by stochastic differential equations based on the continuum approximation by assuming a sufficiently large number of molecules. It has been, however, pointed out that a very low concentration of chemical species often plays crucial roles in a cell [6]. This fact suggests that the continuum approximation is not suitable for describing intracellular reaction dynamics governed by a few molecules. From this point of view, recent theoretical works on catalytic reaction networks (CRNs) have treated molecules discretely and predicted interesting phenomena that cannot be explained by the continuum approximation [7-10]. According to this approach, Awazu and Kaneko [10] have shown that the catalytic reaction dynamics in a $\mathrm{CRN}$ with a homogeneous random-graph topology exhibits self-organized criticality [19-21]. They demonstrated that the size distribution of intermittent chain reactions obeys a power law with the exponent $4 / 3$. This exponent has been explained by regarding the time evolution of the number of reactions as a onedimensional random walk. The other type of studies aims to explore structural features of actual metabolic networks [11-18]. These studies have revealed that most of the metabolic networks possess the scale-free property, i.e., the probability $P(k)$ of a node to have $k$ edges (degree $k$ ) is proportional to $k^{-\gamma}$ for large $k$ [11]. This implies that metabolic networks are highly inhomogeneous in the sense of the degree distribution.

\footnotetext{
*akitomo0416watanabe@eng.hokudai.ac.jp

†yakubo@eng.hokudai.ac.jp
}

In addition, community structures [12-15] and degree-degree correlations [16,17] have been found in many metabolic networks, which are also structural inhomogeneity of CRNs.

Although dynamical and structural properties of CRNs have been extensively studied independently of each other, it is less understood how the network topology affects the reaction dynamics (or vice versa). A qualitative difference in inhomogeneity of networks is supposed to drastically change dynamical properties. It is, therefore, important to clarify the interplay between network structures and reaction processes. In this paper, we study the influence of various types of inhomogeneities in CRNs on the universality class of SOC found by Awazu and Kaneko [10] in homogeneous networks. Surprisingly, our numerical results show that the universality class of SOC is independent of the presence or absence of structural inhomogeneities, such as the scale-free property, community structures, or degree correlations. In a functionally inhomogeneous case where the number of chemical reaction paths catalyzed by an enzyme obeys a power-law distribution, however, the exponent of the avalanche-size distribution deviates from $4 / 3$, which implies the change of the universality class of SOC. We argue these results from the viewpoint of a one-dimensional random-walk model for the time evolution of the number of reactions.

This paper is organized as follows. In Sec. II, after a brief review of the model proposed by Awazu and Kaneko [10], we explain our network models. In Sec. III, we present numerically calculated size distributions of avalanches in the catalytic reaction dynamics in CRNs with various types of inhomogeneities. In addition, the universality class of SOC is discussed based on the random-walk interpretation. Concluding remarks are presented in Sec. IV.

\section{INTRACELLULAR CATALYTIC REACTIONS}

\section{A. Catalytic reaction dynamics}

We study numerically how the inhomogeneity of a CRN affects the universality class of SOC found by Awazu and Kaneko [10]. Thus, we first explain their model describing intracellular catalytic reactions. A CRN is a graph including 


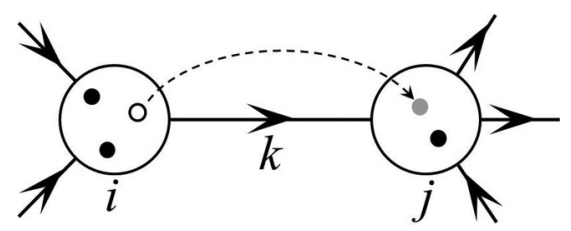

FIG. 1. Schematic representation of reaction dynamics in a CRN. A single chemical reaction from the substrate $i$ to the product $j$ catalyzed by the catalyst $k$ can be considered as molecular transport from the node $i$ to $j$ along the directed edge labeled by $k$.

$N$ nodes and $M$ directed edges. Each node represents a chemical species in a cell, which plays the roles of a substrate, product, and catalyst. Directed edges indicate chemical reaction paths. We assign a node number (catalyst number) to each of the edges. A directed edge from the node $i$ to $j$ with the catalyst number $k$ represents a chemical reaction from the substrate $i$ to the product $j$ catalyzed by the catalyst $k$. Although a single catalyst number is allowed to be assigned to plural edges, considering actual reactions, the catalyst number of an edge from $i$ to $j$ can be neither $i$ nor $j$ and all outgoing edges from a node must have different catalyst numbers. Furthermore, we specify a single node $i_{\mathrm{e}}$ among all nodes as the elimination node from which molecules are eliminated. Catalyst numbers must be selected from node numbers other than $i_{\mathrm{e}}$. Note that networks contain no self-loops and multiple edges with the same direction. For simplicity, a network is assumed to consist of a single strongly connected component.

A network explained above just provides a static reaction map. Dynamical processes of catalytic reactions are actually played by molecules of each chemical species. Representing a single chemical reaction from the substrate $i$ to the product $j$ catalyzed by the catalyst $k$ by transport of a molecule in the node $i$ to $j$ as illustrated by Fig. 1, a dynamical state is specified by the set $\left\{n_{1}, n_{2}, \ldots, n_{N}\right\}$, where $n_{i}$ is the number of molecules contained in the node $i$. Such dynamical states are classified into active and inactive states. In an active state, there is at least one reaction path whose substrate and catalyst molecules both exist, that is, reactions can occur in this state. On the contrary, the state is called inactive if $n_{i} n_{k}=0$ for the substrate $i$ and catalyst $k$ of any reaction path.

A typical series of catalytic reactions starts from inflow of a molecule from the outside of the CRN. This molecule alters its species many times by chemical reactions and is finally eliminated from the cell. These three stages (inflow, reaction, and outflow) are realized as follows. The initial dynamical state at $t=0$ is set as $n_{i}=0$ for any $i(=1,2, \ldots, N)$. At each time step $(t>0)$, we perform the following algorithm: (i) With the probability $q / N$, a molecule is added in a randomly chosen node (inflow), where $q$ is a constant representing the inflow rate. (ii) If the state is active, $\mu n_{\text {tot }}$ molecular pairs are randomly selected, where $n_{\text {tot }} \equiv \sum_{i=1}^{N} n_{i}$ is the total number of molecules in the system and $\mu$ is a constant characterizing the chemical reaction rate, and we move a substrate molecule to the product node if the selected molecular pair consists of the substrate and catalyst of a reaction edge (reaction). (iii) If molecules are moved to the elimination node $i_{\mathrm{e}}$, the molecules are deleted immediately (outflow). Repeating the procedure (i)-(iii) many times, the system reaches the steady state where $n_{\text {tot }}$ does not increase anymore and fluctuates around the steady state value. Reaction processes after reaching the steady state occur intermittently if the inflow rate $q$ is not too large compared to the chemical reaction rate controlled by the parameter $\mu$. We define the size $S$ of an avalanche of chain reactions after the steady state as the number of reactions during a time period of consecutive active states. The avalanche-size distribution function $R(S)$ is calculated by measuring $S$ over a long time after reaching the steady state.

Awazu and Kaneko [10] have calculated $R(S)$ for directed Erdős-Rényi random graphs and found that $R(S)$ obeys a power law,

$$
R(S) \propto S^{-\tau},
$$

where $\tau=4 / 3$. This indicates that the catalytic reaction dynamics exhibits SOC. The value of the exponent $\tau$ identifying the universality class of SOC does not change by varying the inflow rate $q$ and the average in- and out-degree $\left\langle k_{\text {in/out }}\right\rangle$, if $q$ is sufficiently small and $k_{\mathrm{c}}<\left\langle k_{\text {in/out }}\right\rangle \ll N$, where $k_{\mathrm{c}}$ is the degree above which most of the random graphs are connected. Although their stimulating results give deep insights into the catalytic reaction dynamics, the random-graph topology is inadequate to describe inhomogeneous metabolic networks. It is crucial to elucidate how various types of inhomogeneities in CRNs affect SOC.

\section{B. Models of inhomogeneous catalytic reaction networks}

In order to clarify the effect of the inhomogeneity, we deal with several simplified inhomogeneous network models emphasizing features of real-world metabolic networks. The models treated in this paper are summarized below.

\section{Scale-free network}

To extract the scale-free property of metabolic networks, we employ the directed configuration model $[22,23]$. The degree distribution functions are given by $P_{\text {in }}\left(k_{\text {in }}\right)=C_{\text {in }} k_{\text {in }}{ }^{-\gamma_{\text {in }}}$ and $P_{\text {out }}\left(k_{\text {out }}\right)=C_{\text {out }} k_{\text {out }}{ }^{-} \gamma_{\text {out }}$ for $k_{\text {in/out }} \in\left[k_{\text {min }}, \infty\right)$, where $k_{\text {in }}$ and $k_{\text {out }}$ are the in- and out-degree of a node, respectively, $C_{\text {in/out }}$ is the normalization constant, $k_{\min }$ is the minimum degree, and $\gamma_{\text {in/out }}$ is the exponent characterizing the inhomogeneity in the degree distribution. Here, we assume $\gamma_{\text {in }}$ and $\gamma_{\text {out }}$ to be the same, i.e., $\gamma_{\text {in }}=\gamma_{\text {out }}=\gamma$. Thus, the distribution functions of $k_{\text {in }}$ and $k_{\text {out }}$ are commonly described by $P\left(k_{\text {in/out }}\right)=C k_{\text {in/out }}^{-\gamma}$, where $C=1 / \sum_{k=k_{\min }}^{\infty} k^{-\gamma}$. In this model, at first, values of $k_{\text {in }}$ and $k_{\text {out }}$ are assigned to each node according to the degree distribution function, and $k_{\text {in }}$ in-stubs and $k_{\text {out }}$ out-stubs are attached to each node. The network is formed by connecting each of the in-stubs to a randomly chosen out-stub without making multiple edges or loops. Networks with large $N$ formed by this model do not have community structures nor degree correlations. We should note that the average in-degree $\left\langle k_{\text {in }}\right\rangle$ is equal to the average out-degree $\left\langle k_{\text {out }}\right\rangle$ and is uniquely determined by a set of $\gamma$ and $k_{\text {min }}$.

\section{Networks with a community structure and degree correlations}

Community structures found in metabolic networks [1215] are modeled by the following simplification. First, we prepare $v_{\mathrm{c}}$ directed random regular graphs which are 
disconnected from each other. In each of these regular graphs, $n$ nodes are connected by directed edges and both of the in-degree $k_{\text {in }}$ and out-degree $k_{\text {out }}$ of all nodes are equal to $k$, where $1 \ll k<n$. Then, every pair of regular graphs is interconnected by two directed edges bidirectionally. To this end, we randomly choose two nodes $i$ and $i^{\prime}$ from a pair of random regular graphs $v$ and $v^{\prime}$, respectively, and these nodes are connected by an edge directed from $i$ to $i^{\prime}$. Furthermore, the nodes $j$ and $j^{\prime}$ are again randomly selected, respectively, from $v$ and $v^{\prime}$, which are connected by an edge directed from $j^{\prime}$ to $j$. The desired network is formed by repeating this procedure for any pair of regular graphs. If the number of edges in a random regular subgraph $g$ is much larger than the number of intersubgraph edges to and from $g$, namely, $n k \gg 2\left(v_{\mathrm{c}}-1\right)$, the network has a definite community structure. In fact, if we regard node sets of regular subgraphs as communities and ignore directions of edges, the modularity $Q$ [24] that is a widely accepted measure of community structure is rigorously calculated as

$$
Q=\frac{n k}{n k+v_{\mathrm{c}}-1}-\frac{1}{v_{\mathrm{c}}} .
$$

This modularity becomes close to unity when $n k \gg 2\left(v_{\mathrm{c}}-\right.$ 1) $\gg 1$, which implies that the network surely has a community structure.

Degrees of adjacent nodes in actual metabolic networks are generally correlated [16,17]. Among many possible types of degree correlations, the assortative and disassortative mixing have been extensively studied [25-27]. In a network with assortative mixing, nodes with similar degrees are more likely to be connected to each other, while highly connected nodes (hubs) tend to be connected to nodes having few connections in a network with disassortative mixing. Although most of the metabolic networks show disassortative mixing [16,17], we treat a network model exhibiting assortative mixing, because assortative networks can be easily formed by slightly modifying the above community network model and the effect of degree correlations on SOC might be independent of whether mixing is assortative or disassortative. In order to make an assortatively mixed network, we also prepare $v_{\mathrm{c}}$ directed random regular graphs as in the case of the community network model. The only difference is that nodes belonging to different regular graphs have different degrees, that is, nodes in the $v$ th regular graph have the degree $k_{v}$ and $k_{v} \neq k_{v^{\prime}}$ if $v \neq v^{\prime}$. These regular graphs are then connected to each other by two directed edges in the same manner as the community network model. It is obvious from the definition that networks formed by this algorithm show assortative mixing.

\section{Inhomogeneous catalytic functionality}

In addition to structural inhomogeneities in CRNs, it is important to consider the inhomogeneity in catalytic functionality. Before arguing inhomogeneous catalytic functionality, we start with an explanation of the homogeneous networks in the sense of catalytic functionality, which have been adopted in Ref. [10]. In this case, we assign a chemical species (node number) uniformly at random to an edge as a catalyst of the reaction. The probability $H(m)$ of a chemical species to catalyze $m$ kinds of reactions, namely, the probability distribution function of the number of chemical reaction paths catalyzed by a single chemical species, is approximately given by the binomial function

$$
H(m)=\left(\begin{array}{l}
M \\
m
\end{array}\right) \frac{1}{(N-3)^{m}}\left(1-\frac{1}{N-3}\right)^{M-m},
$$

where $N$ and $M$ are the numbers of nodes and edges in the CRN, respectively. The reason why $N-3$ instead of $N$ appears in the above expression is due to the fact that the chemical species corresponding to the substrate, the product, and the elimination node cannot be a catalyst. Since the above distribution function $H(m)$ is derived by ignoring the constraint that the same catalyst number cannot be assigned to plural outgoing edges from a node, $H(m)$ given by Eq. (3) is finite for $N-2<m \leqslant M$ whereas actual $m$ cannot be larger than $N-2$ because of this constraint. The distribution function $H(m)$ gives the average $\langle m\rangle=M /(N-3)$ and decays exponentially for $m \gg\langle m\rangle$. Therefore, every chemical species catalyzes almost the same number of reaction paths.

In actual CRNs, however, some chemical species catalyze only a few reactions, such as urease that hydrolyzes urea to $\mathrm{CO}_{2}$ and ammonia, whereas some catalysts, such as alcohol dehydrogenase responsible for the oxidation of many kinds of primary and secondary alcohols, act on a large number of chemical reaction paths. It is thus crucial to clarify how the inhomogeneous distribution of $m$ affects SOC in the catalytic reaction dynamics. To realize the inhomogeneous distribution of $m$, we randomly choose a catalyst number $i\left(\neq i_{\mathrm{e}}\right)$ according to the probability $p_{i}$ proportional to $i^{-\sigma^{\prime}}$ and assign the number $i$ to a randomly selected edge whose end points are not equal to $i$, where $\sigma^{\prime}$ is a positive exponent. The catalyst for a reaction path from a substrate must not be the chemical species already assigned to outgoing edges from the substrate. This procedure is repeated until catalyst numbers are assigned to all edges. If the condition $N(N-1) / 2 \gg M \gg N$ is satisfied, the number of reaction paths catalyzed by a single chemical species obeys the power law

$$
H(m) \propto m^{-\sigma}
$$

for $m \gg 1$, where the exponent $\sigma$ is related to $\sigma^{\prime}$ via the relation $\sigma=1+1 / \sigma^{\prime}$.

\section{AVALANCHE-SIZE DISTRIBUTION}

\section{A. Avalanche-size distribution for structurally inhomogeneous networks}

We numerically calculated the avalanche-size distribution functions $R(S)$ for scale-free networks, a network with a community structure, and a degree correlated network. For this purpose, the time evolution of the total number of reactions $r(t)$ is first computed according to the algorithm described in Sec. II A. We then find the longest time periods during which dynamical states are all active (active periods). The avalanche size $S$ in an active period is calculated by $S=\sum_{t=t_{\mathrm{i}}}^{t_{\mathrm{f}}} r(t)$, where $t_{\mathrm{i}}$ and $t_{\mathrm{f}}$ are the initial and final times of the active period. In calculations for all these network structures, except for the results in Fig. 3, the number of nodes is fixed at $N=1000$, and the average degrees are set to be as close as possible to $\left\langle k_{\text {in }}\right\rangle=\left\langle k_{\text {out }}\right\rangle=18$, which are the same conditions employed in the previous work [10]. Although average degrees 
of real metabolic networks are usually less than 10 [11], we employed a larger value of $\left\langle k_{\text {in/out }}\right\rangle$ than its realistic value. This is because the computation time becomes long if we use a small value of $\left\langle k_{\text {in/out }}\right\rangle$. Since $R(S)$ is not sensitive to $\left\langle k_{\text {in/out }}\right\rangle$ as shown later, our choice of $\left\langle k_{\text {in }}\right\rangle=\left\langle k_{\text {out }}\right\rangle=18$, which makes it easier to compare our results to those of Ref. [10], is not inadequate to represent statistical properties of catalytic reaction dynamics in real metabolic networks. The inflow rate $q$ and the parameter $\mu$ determining the reaction rate are chosen as $q=3.0 \times 10^{-4}$ and $\mu=40.0$. In order to exclude the effect of inhomogeneous catalytic functionality, catalytic numbers assigned to edges are homogeneously distributed according to Eq. (3). Avalanche sizes are measured during the period from $t=10^{6}$ to $10^{8}$, where $t=10^{6}$ is much larger than the time to reach the steady state. For scale-free networks, we calculated $R(S)$ for the exponents $\gamma=4.0,3.5,3.0,2.8$, and 2.7. Values of the minimum degree $k_{\min }$ for these networks are chosen as $k_{\min }=13,11,10,9$, and 8 , which give $\langle k\rangle=18.78$, $17.53,19.03,19.16$, and 18.26 , respectively. In the case of the community network, we construct a network by setting $v_{\mathrm{c}}=5$ and $n=200$, i.e., five directed random regular graphs of $k_{\text {in }}=k_{\text {out }}=18$ containing 200 nodes are weakly connected to each other. The degree correlated network is formed also by $v_{\mathrm{c}}=5$ and $n=200$, but the degrees of five regular graphs are set as $k_{1}=14, k_{2}=16, k_{3}=18, k_{4}=20$, and $k_{5}=22$. Considering the intersubgraph edges, the average degree of the whole network is $\left\langle k_{\text {in }}\right\rangle=\left\langle k_{\text {out }}\right\rangle=18.02$.

Numerically calculated $R(S)$ 's are presented in Fig. 2. For comparison, $R(S)$ for the directed Erdős-Rényi random

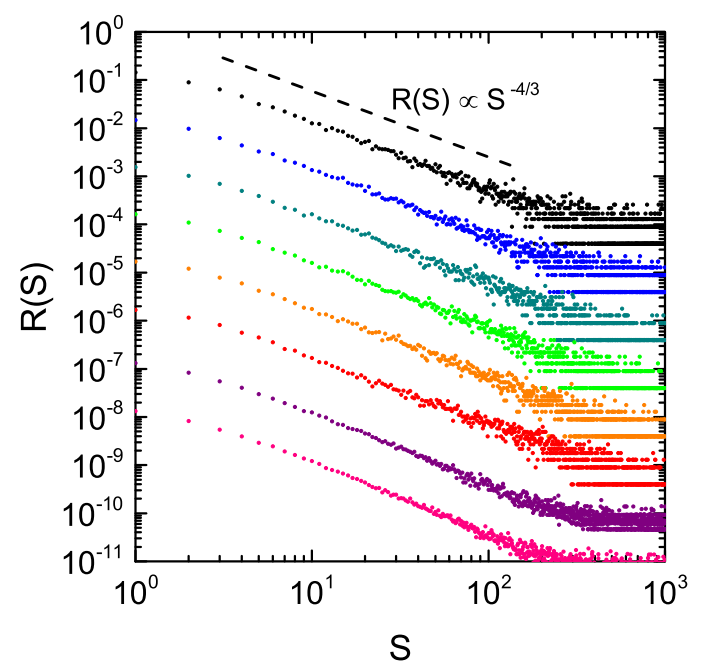

FIG. 2. (Color online) Avalanche-size distributions $R(S)$ for the catalytic reaction dynamics in structurally inhomogeneous CRNs. The top data represent the result for a directed Erdős-Rényi random graph. Results from the second to the sixth from the top show $R(S)$ 's for scale-free networks with the exponents $\gamma=4.0,3.5,3.0,2.8$, and 2.7 , respectively. The second from the bottom and the bottom results give the distribution of $S$ for networks with community structure and degree correlations, respectively, for each of which results are averaged over five samples. The results are vertically shifted for graphical reasons. Details of the network structures and conditions for calculations are described in the main text. The dashed line indicating the slope of $S^{-4 / 3}$ is a guide to the eye.

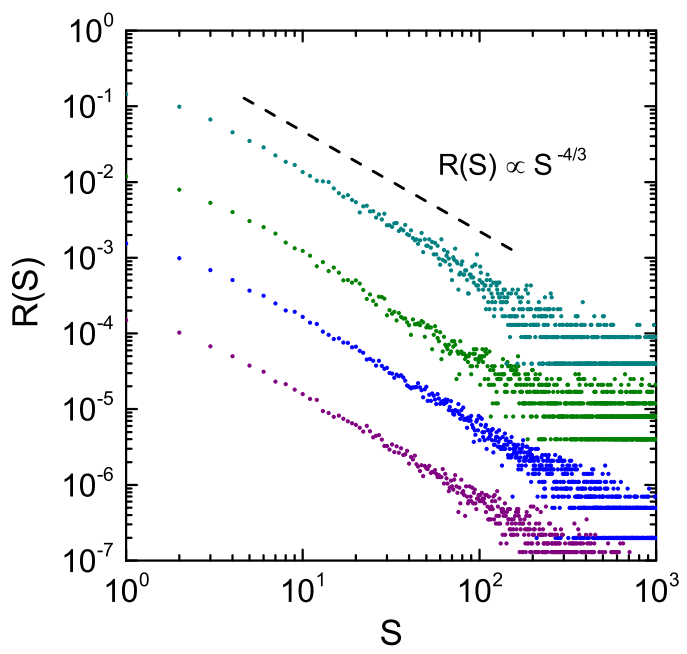

FIG. 3. (Color online) Avalanche-size distributions $R(S)$ for the catalytic reaction dynamics in scale-free CRNs with $\gamma=3.5$ and with various values of parameters characterizing the dynamics. The results from the top to the bottom are calculated for $N=500,\left\langle k_{\text {in }}\right\rangle=$ $\left\langle k_{\text {out }}\right\rangle=35.65, q=6.0 \times 10^{-4}$, and $\mu=80.0$, respectively. Other parameters not specified are the same as those for Fig. 2. The results are vertically shifted for graphical reasons. The dashed line indicating the slope of $S^{-4 / 3}$ is a guide to the eye.

graph is also indicated. In the directed random graph, every node is connected by outgoing edges to each of $N-1$ nodes with the probability $p$, where $N=1000$ and $p=2 / 111$, which gives $\left\langle k_{\text {in }}\right\rangle=\left\langle k_{\text {out }}\right\rangle=18.0$. The distribution $R(S)$ for the Erdős-Rényi random graph shown in Fig. 2 is proportional to $S^{-4 / 3}$, as reported previously by Ref. [10]. Other results in Fig. 2 strongly suggest that $R(S)$ 's for various types of structurally inhomogeneous networks are also proportional to $S^{-4 / 3}$, which implies that the universality class of SOC in the catalytic reaction dynamics is not influenced by structural inhomogeneity. Considering that many of the critical phenomena on scale-free networks alter their universality classes if $\gamma$ changes [28,29], this is a remarkable consequence. It is meaningful to show the robustness of the universality class against the choice of the parameters characterizing the dynamics. Figure 3 exhibits several results of $R(S)$ for different values of $N,\left\langle k_{\text {in/out }}\right\rangle, q$, and $\mu$ from those for Fig. 2 . We see that the universality class of SOC depends on neither structural inhomogeneity nor these parameters [30]. The origin of the robust universality class to structural inhomogeneity will be argued later.

\section{B. Avalanche-size distribution for networks with inhomogeneous catalytic functionality}

Next, we show numerically calculated $R(S)$ 's for CRNs with inhomogeneous catalytic functionality (ICF). In order to distinguish the effect of ICF from that by the structural inhomogeneity, we study the catalytic reaction dynamics on directed Erdős-Rényi random graphs of $N=500$ and $\left\langle k_{\text {in }}\right\rangle=\left\langle k_{\text {out }}\right\rangle=18.0$. The catalyst numbers are assigned to edges so that the number of reaction paths catalyzed by a single chemical species obeys Eq. (4) with $\sigma=2.5$ and 1.5. Other conditions for calculating $R(S)$ are the same as those for Fig. 2. 


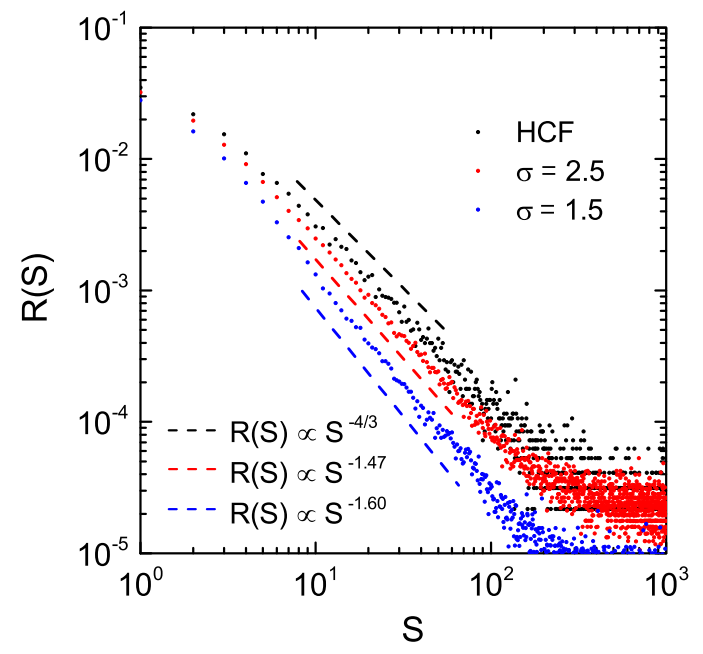

FIG. 4. (Color online) Avalanche-size distributions $R(S)$ for the catalytic reaction dynamics in directed Erdős-Rényi random graphs with inhomogeneous catalytic functionality. The second and third results from the top show $R(S)$ 's for CRNs in which the number of reaction paths catalyzed by a single chemical species is distributed according to Eq. (4) with $\sigma=2.5$ and 1.5, respectively. Results are averaged over five samples. For comparison, we again show the result for a network with a random-graph topology and with homogeneous catalytic functionality at the top, which is the same data shown at the top of Fig. 2. The results are vertically shifted for graphical reasons. Dashed lines indicate the slopes of $S^{-4 / 3}, S^{-1.47}$, and $S^{-1.60}$ from the top to the bottom, respectively.

Our numerical results are shown in Fig. 4. The result for the CRN with homogeneous catalytic functionality (HCF) is also indicated in this figure for comparison, which is the same data as shown at the top of Fig. 2. The results clearly demonstrate that $R(S)$ follows a power law even if catalyst numbers are inhomogeneously assigned to reaction paths (edges). However, the exponent $\tau$ in Eq. (1) for $\sigma=2.5$ or 1.5 deviates from $4 / 3$, which is the value of $\tau$ for the case with HCF. The least-squares fits to the data in the range of $10 \leqslant S \leqslant 100$ give $\tau=1.47 \pm 0.02$ for $\sigma=2.5$ and $\tau=1.60 \pm 0.02$ for $\sigma=$ 1.5. This elucidates that the inhomogeneity in the catalytic functionality changes the universality class of SOC from that for the HCF case.

Let us discuss the change in the universality class of SOC. The exponent $\tau=4 / 3$ for random graphs with HCF has been explained by assuming that the time evolution of the total number of reactions $r(t)$ occurring at a time $t$ behaves as a trajectory of a one-dimensional random walk [10,31]. Under this assumption, since the time duration $D$ of a consecutive avalanche is nothing but the first return time of a random walk, the distribution function of $D$ is proportional to $D^{-3 / 2}$. Using the relation $S \propto D^{3 / 2}$ from $S \sim \int_{0}^{D} \sqrt{\left\langle r^{2}(t)\right\rangle} d t$, we have $R(S) \propto S^{-4 / 3}$ [10]. The assumption of the random-walk behavior of $r(t)$ is supported by the following consideration. The number of reactions $r(t)$ at time $t$ is proportional to the number of combinations of molecules of substrates and their corresponding catalysts at time $t-1$. The number of molecules of a substrate (catalyst) is determined by the numbers of molecules of chemical species responsible for

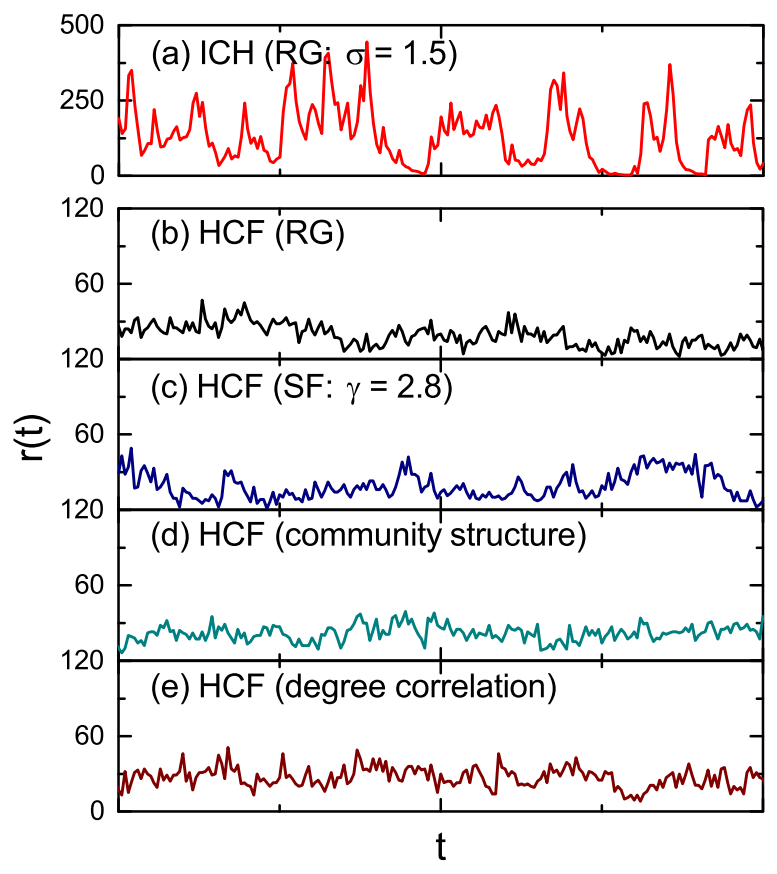

FIG. 5. (Color online) Time evolutions of the number of reactions $r(t)$ during certain periods with 200 time steps included in single consecutive avalanches (a) for a directed Erdős-Rényi random graph with inhomogeneous catalytic functionality $(\sigma=1.5)$ and (b)-(e) for several networks with homogeneous catalytic functionality. Network structures for (b)-(e) are a directed random graph, a scale-free network with the exponent $\gamma=2.8$, a network with community structure, and a network with degree correlations, respectively.

reactions to and from the substrate (catalyst). Furthermore, numbers of molecules of such responsible chemicals are also determined in a similar way, and so on. If catalysts are statistically equivalent as in the case of HCF, these interdependent and complicated nonlinear relations between numbers of molecules of chemical species at time $t$ and $t-1$ make $r(t)$ and $r(t-1)$ uncorrelated, which allows us to regard the profile of $r(t)$ as a one-dimensional random-walk trajectory.

It is thus natural to consider that the change in the universality class is caused by the difference in statistical properties of $r(t)$ for the ICF case from those for the HCF case. Figure 5 shows typical profiles of $r(t)$ during certain time periods included in single consecutive avalanches for a directed Erdős-Rényi random graph with ICF $(\sigma=1.5)$ and for several CRN structures with HCF. It should be noted that the behaviors of $r(t)$ for HCF are almost the same in a statistical sense. On the other hand, $r(t)$ for ICF fluctuates with widely distributed stride lengths. In order to show such characteristics more clearly, we calculate distribution functions of the stride length in $r(t)$. Open and filled symbols in Fig. 6 show the probability distribution functions of one-step change $\Delta r \equiv r(t)-r(t-1)$ in the reaction number corresponding to the stride length of the one-dimensional walk for the ICF and HCF cases, respectively. The results show that the distribution function $F(\Delta r)$ for the networks with HCF decays exponentially, while $F(\Delta r)$ for the ICF case has a long tail. In fact, data presented by open symbols (for $15 \leqslant \Delta r \leqslant 200$ ) 


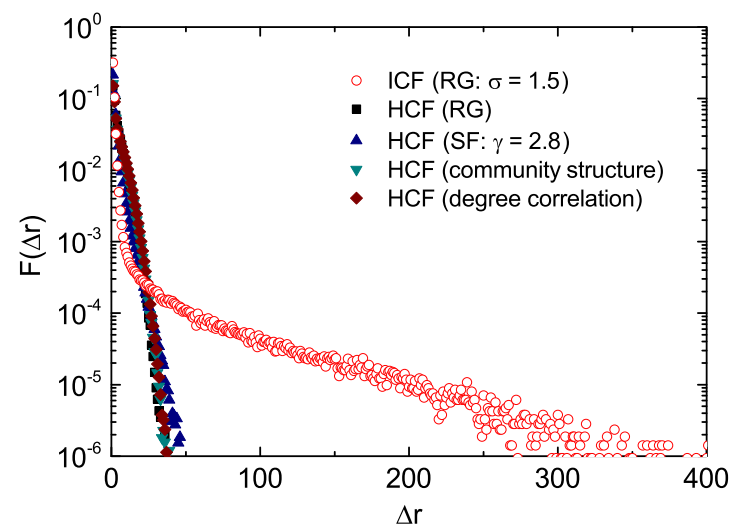

FIG. 6. (Color online) Distribution functions $F(\Delta r)$ of one-step change $\Delta r=r(t)-r(t-1)$ in the reaction number. Open circles represent the result for the catalytic reaction dynamics in a directed Erdős-Rényi random graph with inhomogeneous catalytic functionality $(\sigma=1.5)$. Filled symbols show results for various networks with homogeneous catalytic functionality. Squares, triangles, inverse triangles, and diamonds give $F(\Delta r)$ 's for a random graph, a scale-free network with the exponent $\gamma=2.8$, a network with community structure, and a network with degree correlations. The structures of these networks are the same as those for Figs. 2 and 4.

are well fitted by the strongly stretched exponential function $F(\Delta r) \sim \exp \left[-(\Delta r / 0.15)^{0.27}\right]$. Therefore, $r(t)$ for ICF cannot be regarded as, at least, a simple random walk because of large fluctuations in stride length, whereas stride lengths of $r(t)$ for $\mathrm{HCF}$ are almost constant even for structurally inhomogeneous networks. An extraordinarily large $\Delta r$ for ICF appears when a molecule is added in a vacant node that catalyzes a large number of reaction paths. In contrast, a single-molecule addition into, for example, a vacant hub node in a scale-free network does not trigger many reactions unless the hub node catalyzes a large number of reaction paths.

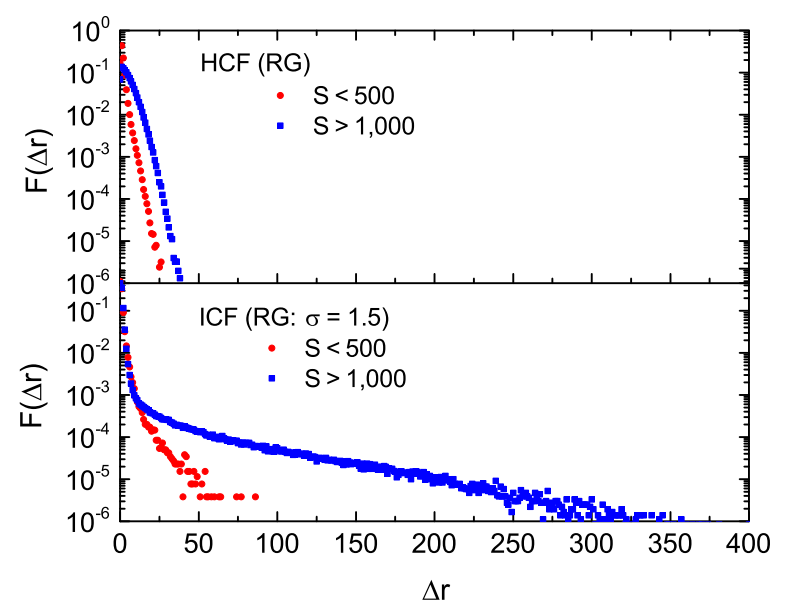

FIG. 7. (Color online) Distribution functions $F(\Delta r)$ of one-step change $\Delta r=r(t)-r(t-1)$ in the reaction number during time periods of small $(S<500)$ and large $(S>1000)$ avalanches. The upper and lower panels indicate the results for directed Erdős-Rényi random graphs with homogeneous and inhomogeneous $(\sigma=1.5)$ catalytic functionality, respectively.
Furthermore, we investigated the correlation between $F(\Delta r)$ and the avalanche size $S$. Figure 7 indicates the distribution functions of $\Delta r$ in time periods including small $(S<500)$ and large $(S>1000)$ avalanches. In the HCF case (upper panel), two distribution functions for $S<500$ and $S>1000$ have almost the same profiles, whereas in the ICF case with $\sigma=1.5$ (lower panel) $F(\Delta r)$ for large avalanches is much broader than that for small avalanches. Although only the results for the random-graph topology are presented in Fig. 7, we obtained similar results for structurally inhomogeneous networks. The fact that $F(\Delta r)$ strongly depends on the avalanche size implies that stride lengths of $r(t)$ for ICF are correlated to the scale of the avalanche, that is, the one-dimensional walk $r(t)$ is not a Markovian process. Thus, $r(t)$ for ICF cannot be considered as a trajectory of a one-dimensional random walk, and the random-walk model leading to $\tau=4 / 3$ is not applicable to the catalytic reaction dynamics with ICF.

\section{CONCLUSION}

We have numerically studied the chemical reaction dynamics in CRNs with various inhomogeneities found in actual metabolic networks. It has been revealed that the distribution function $R(S)$ of the avalanche size $S$ in intermittent chain reactions obeys a power law for all CRNs we dealt with, i.e., $R(S) \propto S^{-\tau}$ for $S \gg 1$. This means that SOC found by Ref. [10] in the reaction dynamics in CRNs with homogeneous random-graph topology can be also observed in inhomogeneous CRNs. In particular, the exponent $\tau$ characterizing the universality class of SOC was found to be $4 / 3$ independently of the structural inhomogeneity such as the scale-free property, community structures, and degree correlations, if the number of reaction paths catalyzed by a single chemical species is almost constant (homogeneous catalytic functionality). This value $4 / 3$ is equal to the exponent $\tau$ for structurally and catalytic-functionally homogeneous networks. Therefore, the universality class of SOC is not influenced by the structural inhomogeneity. For catalyticfunctionally inhomogeneous CRNs, however, the exponent $\tau$ depends on the parameter $\sigma$ that represents how strongly inhomogeneous the distribution of the number of reaction paths catalyzed by a single chemical species is. It has also been argued that the change in the universality class of SOC in the case of inhomogeneous catalytic functionality is caused by the fact that the time evolution $r(t)$ of the number of reactions at time $t$ cannot be regarded as a trajectory of a one-dimensional random walk because stride lengths of $r(t)$ for inhomogeneous catalytic functionality are correlated to the avalanche size. This is in contrast to the case of homogeneous catalytic functionality in which the similarity between $r(t)$ and a trajectory of a simple random walk leads to $\tau=4 / 3$.

Although our numerical results demonstrate the influence of the inhomogeneity in catalytic functionality to the universality class of SOC, it is still an open question why the catalytic reaction dynamics under ICF exhibits SOC in spite of the lack of the theoretical background by the random-walk model. The present result shown in Fig. 4 suggests that the exponent $\tau$ seems to be a decreasing function of $\sigma$. This implies that 
the stronger the inhomogeneity in catalytic functionality the more homogeneous the avalanche-size distribution becomes. In order to clarify this strange behavior, it is crucial to elucidate the relation between the exponents $\sigma$ and $\tau$. Further investigations including these problems will provide insights into the catalytic reaction dynamics in biological cells.

[1] M. Eigen and P. Schuster, Naturwissenschaften 65, 7 (1978).

[2] P. F. Stadler, W. Fontana, and J. H. Miller, Physica D 63, 378 (1993).

[3] D. Segré, D. Ben-Eli, and D. Lancet, Proc. Natl. Acad. Sci. USA 97, 4112 (2000).

[4] S. Jain and S. Krishna, Proc. Natl. Acad. Sci. USA 98, 543 (2001).

[5] C. Furusawa and K. Kaneko, Phys. Rev. Lett. 90, 088102 (2003).

[6] H. H. McAdams and A. Arkin, Trends Genet. 15, 65 (1999).

[7] N. M. Shnerb, Y. Louzoun, E. Bettelheim, and S. Solomon, Proc. Natl. Acad. Sci. USA 97, 10322 (2000).

[8] Y. Togashi and K. Kaneko, Phys. Rev. Lett. 86, 2459 (2001).

[9] A. Awazu and K. Kaneko, Phys. Rev. E 76, 041915 (2007).

[10] A. Awazu and K. Kaneko, Phys. Rev. E 80, 010902(R) (2009).

[11] H. Jeong, B. Tombor, R. Albert, Z. N. Oltvai, and A.-L. Barabási, Nature (London) 407, 651 (2000).

[12] E. Ravasz, A. L. Somera, D. A. Mongru, Z. N. Oltvai, and A.-L. Barabási, Science 297, 1551 (2002).

[13] R. Guimerà and L. A. N. Amaral, Nature (London) 433, 895 (2005).

[14] M. E. J. Newman, Proc. Natl. Acad. Sci. USA 103, 8577 (2006).

[15] K. Takemoto and S. Borjigin, PLoS ONE 6, e25874 (2011).

[16] S. Maslov and K. Sneppen, Science 296, 910 (2002).

[17] M. E. J. Newman, Phys. Rev. E 67, 026126 (2003).

\section{ACKNOWLEDGMENTS}

This work was supported by a Grant-in-Aid for Scientific Research (Grant No. 25390113) from Japan Society for the Promotion of Science. Numerical calculations in this work were performed in part on the facilities of the Supercomputer Center, Institute for Solid State Physics, University of Tokyo.
[18] C. Song, S. Havlin, and H. A. Makse, Nature (London) 433, 392 (2005).

[19] P. Bak, C. Tang, and K. Wiesenfeld, Phys. Rev. Lett. 59, 381 (1987).

[20] P. Bak and K. Sneppen, Phys. Rev. Lett. 71, 4083 (1993).

[21] H. J. Jensen, Self-Organized Criticality (Cambridge University Press, Cambridge, 1998).

[22] M. E. J. Newman, S. H. Strogatz, and D. J. Watts, Phys. Rev. E 64, 026118 (2001).

[23] E. A. Bender and E. R. Canfield, J. Combinational Theory Ser. A 24, 296 (1978)

[24] M. E. J. Newman and M. Girvan, Phys. Rev. E 69, 026113 (2004).

[25] M. E. J. Newman, Phys. Rev. Lett. 89, 208701 (2002).

[26] M. E. J. Newman, SIAM Rev. 45, 167 (2003).

[27] A.-L. Barabási and Z. N. Oltvai, Nat. Rev. Genet. 5, 101 (2004).

[28] R. Cohen, D. ben-Avraham, and S. Havlin, Phys. Rev. E 66, 036113 (2002).

[29] S. N. Dorogovtsev, A. V. Goltsev, and J. F. F. Mendes, Rev. Mod. Phys. 80, 1275 (2008)

[30] It should be noted that the total number of molecules $n_{\text {tot }}$ keeps growing and the dynamics never reach the steady state if $\left\langle k_{\mathrm{in} / \mathrm{out}}\right\rangle$ is too small. In addition, if $q$ is too large, chemical reactions do not exhibit intermittency. We need to choose $\left\langle k_{\text {in/out }}\right\rangle$ and $q$ within the ranges of $k_{\mathrm{c}} \ll\left\langle k_{\text {in/out }}\right\rangle \ll N$ and $q \ll 1$ to obtain SOC behavior, as pointed out at the end of Sec. II A.

[31] T. Jonsson and J. F. Wheater, arXiv:cond-mat/9607087. 